\title{
Hypernuclear physics studies of the PANDA experiment at FAIR
}

\author{
Alicia Sanchez Lorente ${ }^{1, a}$ \\ ${ }^{1}$ Helmholtz Institut Mainz, D-55099 Germany. \\ Tel.: +49 $613139-27381$ \\ Fax: $+49613139-27448$
}

\begin{abstract}
Hypernuclear research will be one of the main topics addressed by the PANDA experiment at the planned Facility for Antiproton and Ion Research FAIR at Darmstadt (Germany). [1,2] Thanks to the use of stored $\bar{p}$ beams, copious production of double $\Lambda$ hypernuclei is expected at the PANDA experiment, which will enable high precision $\gamma$ spectroscopy of such nuclei for the first time, and consequently a unique chance to explore the hyperon-hyperon interaction. In particular, ambiguities of past experiments in determining the strength of the $\Lambda \Lambda$ interaction will be avoided thanks to the excellent energy precision of a few $\mathrm{keV}$ (FWHM) achieved by germanium detectors. Such a resolution capability is particularly needed to resolve the small energy spacing of the order of $(10-100) \mathrm{keV}$, which is characteristic from the spin doublet in hypernuclei the so-called "hypernuclear fine structure".

In comparison to previous experiments, PANDA will benefit from a novel technique to assign the various observable $\gamma$-transitions in a unique way to specific double hypernuclei by exploring various light targets. Nevertheless, the ability to carry out unique assignments requires a devoted hypernuclear detector setup. This consists of a primary nuclear target for the production of $\Xi^{-}+\bar{\Xi}$ pairs, a secondary active target for the hypernuclei formation and the identification of associated decay products and a germanium array detector to perform $\gamma$ spectroscopy.

Moreover, one of the most challenging issues of this project is the fact that all detector systems need to operate in the presence of a high magnetic field and a large hadronic background. Accordingly, the need of an innovative detector concept will require dramatic improvements to fulfil these conditions and that will likely lead to a new generation of detectors. In the present work details concerning the current status of the activities related to the detector developments for this challenging programme will be given.

Among these improvements is the new concept for a cooling system for the germanium detector based on a electro-mechanical device. In the present work, the cooling efficiency of such devices has been successfully tested, showing their capability to reach liquid nitrogen temperatures and therefore the possibility to use them as a good alternative to the standard liquid nitrogen dewars. Furthermore, since the momentum resolution of low momentum particles is crucial for the unique identification of hypernuclei, an analysis procedure for improving the momentum resolution in few layer silicon based trackers is presented.
\end{abstract}

\footnotetext{
ae-mail: lorente@kph.uni-mainz.de
} 


\section{Introduction}

The simultaneous production and implementation of two $\Lambda$ particles into a nucleus is intricate. There is a possibility to produce multi-strange hypernuclei in heavy ion collisions via coalescence $[3,4]$. The first observation of antihypernuclei by the STAR collaboration impressively illustrates the potential of this method [5]. However, high resolution spectroscopy of excited states is not feasible. To produce double hypernuclei in a more 'controlled' way the conversion of a captured $\Xi^{-}$and a proton into two $\Lambda$ particles can be used. This process releases, ignoring binding energy effects, only $28 \mathrm{MeV}$. For light nuclei there exists, therefore, a significant probability of the order of a few percent that both $\Lambda$ hyperons are trapped in the same excited nuclear fragment [6-11]. After an atomic cascade, the $\Xi^{-}$hyperon is eventually captured by a secondary target nucleus and converted via the $\Xi^{-} p \rightarrow \Lambda \Lambda$ reaction into two $\Lambda$ hyperons. In a similar two-step process relatively low momentum $\Xi^{-}$can also be produced using antiproton beams in $\bar{p} p \rightarrow \Xi^{-} \bar{\Xi}^{+}$or $\bar{p} n \rightarrow \Xi^{-} \bar{\Xi}^{0}$ reactions if this reactions happens in a complex nucleus where the produced $\Xi^{-}$can re-scatter $[12,13]$. The advantage as compared to the kaon induced reaction is that antiprotons are stable and can be retained in a storage ring. This allows a rather high luminosity even with very thin primary targets. Because of the two-step mechanism, spectroscopic studies, based on two-body kinematics like in single hypernucleus production, cannot be performed. Spectroscopic information on double hypernuclei can therefore only be obtained via their decay products. The kinetic energies of weak decay products are sensitive to the binding energies of the two $\Lambda$ hyperons. While the double pionic decay of light double hypernuclei can be used as an effective experimental filter to reduce the background [13] the unique identification of hypernuclei ground states only via their pionic decay is usually hampered by the limited resolution.

For the sake of clarity, the decay chain which corresponds to the sequential mesonic decay of ${ }_{\Lambda \Lambda}^{10} \mathrm{Be}$ is shown below,

$$
\begin{aligned}
& { }_{\Lambda \Lambda}^{10} \mathrm{Be} \rightarrow{ }_{\Lambda}^{10} B+\pi^{-} \\
& { }_{\Lambda}^{10} B \rightarrow{ }^{10} C+\pi^{-}
\end{aligned}
$$

Instead, $\gamma$-rays emitted via the sequential decay of excited double hypernuclei may provide precise information on the level structure $[14,15]$.

\section{Integration inside the PANDA spectrometer}

This devoted experimental setup consist of,

- A primary target system at the entrance to the central tracking detector of $\bar{P} A N D A$.

- A small secondary active sandwich target composed of silicon detectors and ${ }^{9} \mathrm{Be},{ }^{10,11} \mathrm{~B}$ or ${ }^{12,13} \mathrm{C}$ absorbers to slow down and stop the $\Xi^{-}$and to identify the weak decay products.

- To detect the $\gamma$-rays from the excited double hypernuclei an array of 16 n-type Germanium triple Cluster-arrays will be added. To maximize the detection efficiency the $\gamma$-detectors must be arranged as close as possible to the target at backward axial angles.

A big challenge to be solved in order to place the hypernuclear detector setup, is the limited space available at the entrance of the PANDA spectrometer. Therefore, some modifications of the inner part of the former spectrometer is needed. In particular, due to the fact that double $\Lambda$-Hypernuclei formation proceeds in a two-steps process, a dedicated target system consisting of a primary and secondary interaction point with an associated beam line is required (see Fig. 1). Since this target 

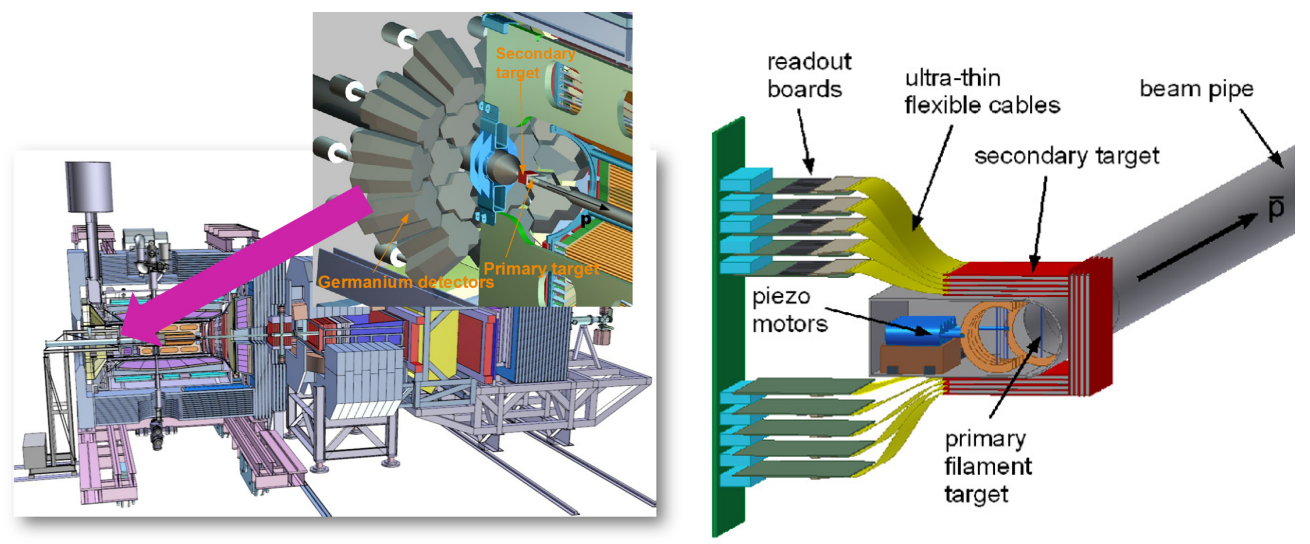

Figure 1. Schematic view of the hypernuclear setup in PANDA. The picture on the left shows a side view of the hypernuclear experimental setup. The picture on the right shows the mechanism which holds the target at a constant interaction rate by an automatic feedback system which continuously adjusts the wire positions via stepping motors. The target mechanism is mounted inside the vertex vessel, surrounded by the moveable parts of the secondary active target(red structure). The principle foreseen to root the analog signals of the sensors outside the interaction region by means of flexible ultra-thin cables is also depicted in this picture.

system has to be placed outside the interaction region of the PANDA spectrometer, detectors such as the Micro Vertex Detector (MVD) and Backward EndCap Calorimeter will be removed to avoid unnecessary radiation damage. In addition, the central frame dedicated to hold the beam line and the central tracker detectors will also be accordingly adapted to the hypernuclear setup.

\section{Primary target}

The main role of the primary target will be the production of low momentum $\Xi$ hyperons. Although heavy targets are more efficient for re-scattering of the produced primary particles and hence for the emission of low momentum $\Xi$ hyperons, light targets will be preferred in order to avoid a high hadronic background into backward axial angles, where the germanium detectors are located. Furthermore, Coulomb scattering in heavy primary targets leads to significant losses of antiprotons. Additionally, it is required that the luminosity of the $\bar{p}$-beam remains as constant as possible. In addition, high interaction rates will be avoided by choosing an appropriate fraction of the beam halo onto the target, what can be achieved by an monitoring mechanism where the beam as well as the target can be steered till the desired interaction rate is reached ( see Fig.1). As a result, the use of thin carbon micro-ribbons [16] seems to be the best candidate to allow a stable operation over a sufficiently long measuring period.

\section{Secondary active target}

The purpose of the active volume of the secondary target is the tracking of charged particles generated during the first and the secondary interaction. As a consequence, its geometry is based on a compact structure where silicon micro-strip detectors layers are in direct contact with absorber material [17]. In analogy to the germanium detectors array, this device has to be able to operate in extreme conditions 
such as a large hadronic environment, since it is close to the interaction point. Furthermore, the material budget on the detector volume must be kept low. The feasibility of such a device has recently been studied in Mainz, by evaluating the influence of putting layers of absorber material directly on a silicon sensor [17]. Results have shown no significant change on the preamplifier signal caused by the vicinity of an boron layer [17]. In order to avoid a huge load of hadronic flux on the readout electronics, the use of Ultra-thin Al-Polyimide micro-cables (see Fig.1) is foreseen, by routing the sensor analogue signals to the readout system outside the interaction region and as a result to decrease the amount of material budget on the detecting volume. Further activities are planned to investigate the
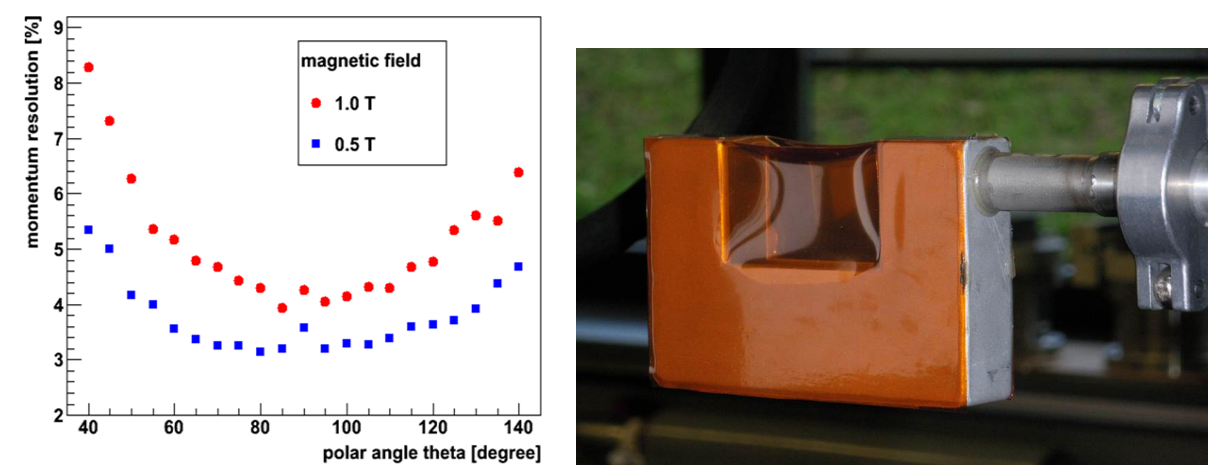

Figure 2. The picture on the left depicts the dependence of the tracking accuracy on the magnetic field strength and the polar angle. On the right panel a first evacuated target chamber prototype is shown. A $75 \mu \mathrm{m}$ Kapton foil was glued to the aluminium frame. During the pumping process a slight modification of the surface under vacuum was observed. After the test the foil remained slightly deformed.

effect of the cable length on the signal transmission are in progress. In addition, the primary target as well as the steering mechanism has to operate embedded in a high vacuum environment. In the design of a beam vacuum chamber, the choice of the geometry, materials and pumping is determined by the compromise between vacuum and mechanical stability requirements on the one side and the necessity to minimize the amount of material between the beam and the secondary active target on the other side. However, the material budget of such a compact structure may result in a poor tracking efficiency. As a consequence, a compromise between the production factor and a good tracking accuracy must be found by means of a Monte-Carlo simulation. According with the current geometry (see Fig. 1), the material budget corresponds to a four composite layers structure surrounding the primary interaction point (two additional layers structures have been added to improve the tracking accuracy).

In the following the complete description of the hypernuclear detector set up was implemented within the PANDARoot [19] simulation framework. The starting point of the Monte-Carlo simulation, is the generation of $200000 \Xi^{-}$hyperons in the momentum range from $100 \mathrm{MeV} / \mathrm{c}$ to $500 \mathrm{MeV} / \mathrm{c}$. From this primary data set only a few percent, namely a 5.2\% is stopped in the absorber material of the secondary active target. Additionally, it is interesting to note that most of the produced hyperons are stopped very close to the beam pipe. Increasing the amount of absorber material to increase the stopping factor will result, nevertheless in a poor tracking efficiency, therefore the necessity to keep the material budget around the beam pipe as low as possible in order to have a good momentum resolution of the decay charged products. Generally, a good tracking efficiency depends on the magnetic field strength, number of tracking detectors and particle momentum. In the case of low momentum pions, emitted from the weak decay of the produced hypernuclei, further modifications of the current tracking 
algorithms have to be accomplished. On one side, the tracking of pions emitted from the hypernuclei decay with a momentum ranging between $70 \mathrm{MeV} / \mathrm{c}$ and $140 \mathrm{MeV} / \mathrm{c}$ will require a reduction of the magnetic field strength.The momentum resolution of low momentum pions have been studied by using the tracking package GenFit [20,21], which additionally takes care of effects due to energy losses and multi-scattering process inside the detector material. For simplicity, the nominal value of $100 \mathrm{MeV} / \mathrm{c}$ for the pion momentum has been chosen to evaluate the tracking accuracy, since pion momentum emitted from a the weak decay of hypernuclei ranges from $70 \mathrm{MeV} / \mathrm{c}$ to $140 \mathrm{MeV} / \mathrm{c}$.

The left panel of Fig. 2 shows details of the simulation and tracking results for pions emitted with a momentum of $100 \mathrm{MeV} / \mathrm{c}$. One can see that the momentum resolution decreases as a function of the polar angle $\theta$, reaching a minimum around $90^{\circ}$.

For instance, the momentum resolution of $100 \mathrm{MeV} / \mathrm{c}$ pions emitted at a fixed polar angle of $70^{\circ}$ amounts $3 \%$ of the total momentum. In addition, it has also been observed that the mean value of the distribution differs from the nominal value of $100 \mathrm{MeV} / \mathrm{c}$. These uncertainties appear due to not optimal error treatment of the energy loss and therefore further improvements of the tracking algorithm for this purpose are in progress.

Other important aspect is to investigate the mechanical stability of the target chamber by means of a first chamber prototype shown in Fig. 2. This Figure shows an aluminium parallelepiped structure $(110 \mathrm{~mm} \times 72 \mathrm{~mm} \times 24 \mathrm{~mm})$ with a thickness of $3 \mathrm{~mm}$. Since the target chamber has to be as transparent as possible to scattered particles close to the interaction point, the chamber has been cut off. This area will be in the following covered by a thin foil. The choice and the thickness of this material has to be also justified. During the test, the chamber glued to a $75 \mu \mathrm{m}$-thick kapton foil was pumped by a roughing pump to $1 \times 10^{-4}$ mbar. As a consequence a slight modification of the surface under vacuum was observed. After the test the foil remained slightly deformed. A better understanding of the mechanical properties of different materials is foreseen, in order to elaborate a chamber design which remains in the elastic range, namely not steady deformations are desirable.
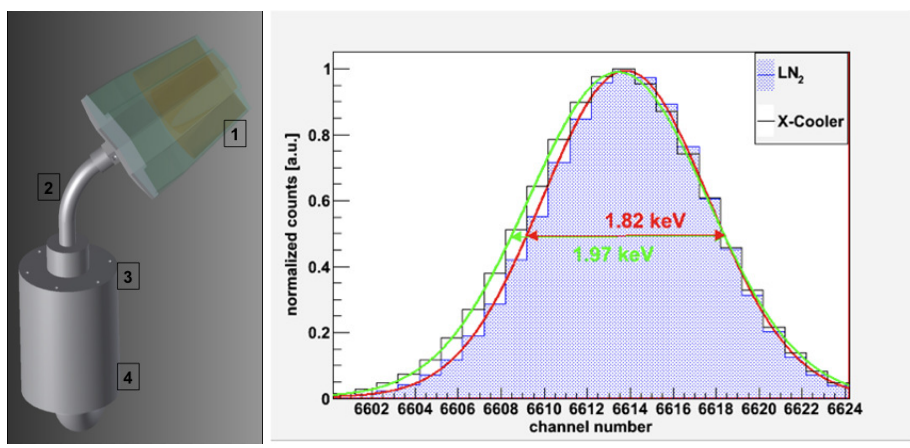

Figure 3. HPGe triple cluster array assembled to a X-Cooler device. The encapsulated n-type coaxial HPGe crystals (EUROBALL) are arranged in a triangular form. The free space behind the crystals is foreseen for electronics. The connection of the crystal vessel to the cold head of the cooler is flexible to enable the placement of each cluster at backward axial angles. (1) encapsulated crystals, (2) flexible neck, (3) X-Cooler cold head, (4) $\mathrm{X}$-Cooler Cable. The figure on the right shows the energy spectra corresponding to the $1,332 \mathrm{keV}$ line of ${ }^{60} \mathrm{Co}$ for two different cooling devices. The dashed spectrum corresponds to the case where the germanium crystal has been cooled electro-mechanically showing a slightly worsening of the energy resolution. 


\section{The HPGe cluster Array}

In order to increase the detection efficiency needed for a high resolution $\gamma$-Spectroscopy, the highpurity germanium (HPGe) detectors array has to be placed as close as possible to the interaction point and cover a wide solid angle. Due to the limited space of the PANDA spectrometer, the arrangement of the germanium detector array will only be possible at backward axial angles. That means for instance, that the operation of these detectors will have to withstand a large flux of hadronic background and a high magnetic field [18], which can influence the good energy resolution $(\sim 3 \mathrm{keV}$ at the $1,332 \mathrm{MeV}$ line of ${ }^{60} \mathrm{Co}$ ) of these detectors. A possible solution for the space limitation has been to replace the standard cooling system, based on liquid nitrogen dewars, by a mechanical cooling device [22]. Fig. 3 shows a prototype for a triple germanium cluster array cooled by an electromechanical device. After installation of each of the encapsulated germanium crystals in the cryostat or vacuum vessel, the system has to reach optimum vacuum conditions to be properly operated.

The cooling efficiency of these devices has been successfully tested for three encapsulated germanium crystals without observing any additional worsening of the energy resolution [23, 24]. Further investigations are currently taking place in Mainz at a dedicated test station. The scope of these studies is to evaluate to what extent the energy resolution of a germanium detector, cooled electromechanically, can be influenced. For this reason, an ORTEC GEM-75205P device and analogue readout electronics has been used. The energy resolution of such a device has been measured with a ${ }^{60}$ Co source considering two different cooling devices, namely a standard liquid nitrogen dewar and a $\mathrm{X}$-Cooler device [22]. For the case of a standard cooling system, the energy resolution was found to be $1.86 \mathrm{keV}$ for $1.332 \mathrm{keV}$ line ${ }^{1}$. The one achieved by the $\mathrm{X}$-cooler device has been $1.97 \mathrm{keV}$, which seems to be consistent. Fig. 3 shows the energy spectra corresponding to the $1.332 \mathrm{keV}$ line of ${ }^{60} \mathrm{Co}$, obtained by considering the two cooling systems named above. The dashed spectrum corresponds to the case where the germanium crystal has been cooled electro-mechanically and although the energy resolution for this case is slightly worse, one can see that the use of the X-Cooler as a cooling option seems to be nevertheless acceptable. Further investigations on temperature effects and their impact on the spectroscopy properties as well as the performance of the detector and the X-Cooler assembly are in progress. In addition, activities concerning the use of digital electronics to evaluate pile-up effects, and radiation damages in a high flux hadronic environment are also under preparation [24].

\section{Conclusions}

Hypersystems provide a link between nuclear physics and QCD, and allow the study the basic properties of strongly interacting systems. Antiproton collisions with nuclei as it is planned at the PANDA experiment is an ideal tool to produce exclusive $\Xi^{-} \bar{\Xi}^{+}$pairs in nuclei at moderate momenta. In addition, the use of a statistical model may help to predict the population of individual excited, particle stable states in double $\Lambda$ hypernuclei. Thanks to the high capability of the $\bar{P}$ ANDA spectrometer the $\gamma$-spectroscopy of double $\Lambda$ hypernuclei seems feasible.

\section{References}

[1] http://www.gsi.de

[2] http://www.gsi.de/fair/.

[3] A. K. Kerman and M. S. Weiss, Phys. Rev. C 8, 408 (1973).

[4] A. S. Botvina and J. Pochodzalla, Phys. Rev. C 76, 024909 (2007).

\footnotetext{
${ }^{1}$ The energy resolution provided by Ortec is about $2.05 \mathrm{keV}$
} 
[5] The STAR Collaboration Science 2010328 58-62.

[6] Y. Yamamoto, M. Sano and M. Wakai, Prog. Theor. Phys. Suppl. 117, 265 (1994).

[7] T. Yamada and K. Ikeda, Phys. Rev. C 56, 3216 (1997).

[8] Y. Yamamoto, M. Wakai, T. Motoba, T. Fukuda, Nucl. Phys. A 625, 107 (1997).

[9] Y. Hirata et al., Nucl. Phys. A 639, 389c (1998).

[10] Y. Hirata et al., Prog. Theor. Phys. 102, 89 (1999).

[11] S. Aoki et al., KEK E176 Collaboration, Nucl. Phys. A 828, 191 (2009).

[12] F. Ferro et al., Nucl. Phys. A789, 209 (2007).

[13] Physics Performance Report for PANDA: Strong Interaction Studies with Antiprotons, arXiv:0903.3905v1.

[14] J. Pochodzalla, Nucl. Phys. A 754, 430c 2005.

[15] A. Sanchez Lorente, A. Botvina and J. Pochodzalla, Phys. Lett. B 697222 (2011).

[16] http://www-panda.gsi.de/db/papersDB/PC19-050217_panda_tpr.pdf

[17] S. Bleser, PhD.Thesis, private communication.

[18] A. Sanchez Lorente et al., Nucl. Inst. Meth A 573, 410(2007).

[19] FairRoot,Simulation and Analysis Framework, http://fairroot.gsi.de.

[20] http://sourceforge.net/projects/genfit.

[21] CERN Program Library W5013 (1991).

[22] ORTEC. X-Cooler ${ }^{\text {TM }}$ II- Mechanical Cooler for HPGe detectors user manual.

[23] M. Wolf, J. Kojouharova, I. Kojouharov et al. Proceedings COMSOL Users Conference.

[24] M. Steinen, Master Thesis, private communication. 